\title{
Post transfusion septicaemia 1980-1989: Importance of donor arm cleansing
}

\author{
A Puckett, G Davison, C C Entwistle, J A J Barbara
}

\begin{abstract}
Aims: To determine the prevalence of Pseudomonas fluorescens on the arms of blood donors, and to elucidate one possible cause for its predominance $(60 \%$ of cases during 1980-89) in exogenous post transfusion septicaemia (PTS).

Methods: Skin swabs were taken from the arms of 782 blood donors and cultured on to heated blood agar. After incubation, Oxidase reagent and the Gram stain were used to select non-fermentative Gram negative rods, which were then subcultured and identified using the Analytical Profile System (API) 20 NE system.

Results: Non-fermentative Gram negative rods were found on the arms of $11.7 \%$ of donors, Pseudomonas spp on $1.0 \%$, and Ps fluorescens on the arms of $0.3 \%$ of donors.

Conclusion: This evidence emphasises the absolute requirement for efficient skin cleansing of blood donors' arms to minimise the risk of exogenous PTS.
\end{abstract}

Post transfusion septicaemia (PTS), once a well recognised hazard of blood transfusion, became less common when glass containers were replaced with a closed plastic pack system for taking blood donations. ${ }^{1}$ Although the incidence of PTS has decreased, it has not disappeared entirely ${ }^{1}$; there seems to have been a resurgence of interest in it and reports about it over the past few years, in both the United Kingdom and the United States of America. ${ }^{23}$ In 1987 there were five reported cases in this country of which four were fatal. This is likely to reflect a greater mortality than from the total of hepatitis B, human immune deficiency virus and syphilis transmitted by transfusion of cellular blood products. ${ }^{4-7}$

Logically, the most likely bacteria to cause

Accepted for publication 31 July 1991

Table 1 Cases of exogenous post transfusion septicaemia in United Kingdom (1980-89)

\begin{tabular}{clll}
\hline Year & Presentation & Organism & Reference \\
\hline 1981 & Death & Pseudomonas fluorescens & Hewitt (personal communication) \\
1982 & Death & Pseudomonas fluorescens & Murray et al \\
1982 & Death & Pseudomonas fluorescens & (Personal communication) \\
1984 & Severe illness & Pseudomonas fluorescens & Murray et al $^{5}$ \\
1986 & Death & Pseudomonas fluorescens & Scott et $^{6}$ \\
1987 & Death & Pseudomonas fluorescens & The Times $^{7}$ \\
1987 & Death & Pseudomonas fluorescens & Napier (personal communication) \\
1987 & Isolation & Pseudomonas fluorescens & Mitchell (personal communication) \\
1987 & Death & Pseudomonas sp & Hewitt (personal communication) \\
1987 & Death & Pseudomonas sp & (Personal communication) \\
1987 & Illness & Staphylococcus epidermidis & Fraser (personal communication) \\
1988 & Illness & Serratia marcescens & Mitchell (personal communication) \\
1988 & Death & Erwinia herbicola & Robinson (personal communication) \\
1989 & Illness & Staphylococcus saprophyticus & Napier (personal communication) \\
\hline
\end{tabular}

PTS would be skin flora (Staphylococcus epidermidis, Micrococcus spp, and, in the deeper layers of the skin, "diphtheroids"), and yet a survey of known cases 1980-89 (table 1) shows that $70 \%$ of them were caused by Pseudomonas spp, $60 \%$ by Ps fluorescens. Whether this is because of the organism's demonstrated propensity for growing in donor blood, ${ }^{1}$ or because of its ubiquity, or both, is not known. To shed light on this, 782 donor arms were swabbed to determine the prevalence of nonfermentative Gram negative rods, especially $P_{s}$ fluorescens.

\section{Methods}

Skin swabs were taken from the arms of 782 blood donors at blood collection sessions around the Oxford region. Sterile cotton wool swabs were soaked in physiological saline before use and then rubbed firmly on the venepuncture site on the donor arm before disinfection. They were cultured on to heated blood agar one to two hours after collection and incubated at $30^{\circ} \mathrm{C}$ in air for 48 hours. After this, they were flooded with $1.0 \% \mathrm{NNN}^{\prime} \mathrm{N}$ " Tetramethyl-p-phenylene- diamine-dihydrochloride (Oxidase reagent). After 10 seconds those colonies turning purple were picked and subcultured on to heated blood agar.

Subcultures were incubated at $30^{\circ} \mathrm{C}$ in air for 48 hours. Once in pure culture, the colonies were Gram stained and the oxidase reaction rechecked.

Those still oxidase positive and Gram negative were set aside for identification with the Analytical Profile Index (API) $20 \mathrm{NE}$ system (La Balme des Grotte, 38390 Montalieu Vercieu, France) for non-fermentative bacteria (table 2).

Only a limited number of API identifications were available, so distinctive, easily recognisable colony types that had been identified on several occasions were assumed to be the same type of organism. This level of identification was considered sufficient within the requirements of this study (table 3 ).

\section{OXFORD CENTRE ARM CLEANSING METHOD}

The venepuncture site is vigorously wiped with a swab containing $0 \cdot 2 \%$ chlorhexidine acetate and $2 \%$ cetrimide (Mediprep, Smith and Nephew, Hull, England), and then a gauze pad, well soaked with Hibitane in spirit $(0.5 \%$ chlorhexidine gluconate in $70 \%$ industrial methylated spirit), is applied for 30 seconds.

Results

The results can be summarised as follows: 
Table 2 Organisms from donor arms identified by API

\begin{tabular}{lc}
\hline Organism & $\begin{array}{l}\text { Number of } \\
\text { times isolated }\end{array}$ \\
\hline Pseudomonas fluorescens & 2 \\
Pseudomonas spp & 6 \\
Flavobacterium spp & 8 \\
Bordetella bronchoseptica & 3 \\
Aeromonas salmoncida & 3 \\
Alcaligenes spp & 1 \\
CDC group IV C-2 & 1 \\
Achromobacter spp & 1 \\
Moraxella/Pseudomonas & 8 \\
Moraxella/Pasturella & 7 \\
Flavobacterium/Pasturella & 3 \\
Flavobacterium/Pseudomonas & 2 \\
Total & 45 \\
\hline
\end{tabular}

Table 3 Putative organisms from donor arms identified by colonial morphology

Moraxella/Pseudomonas

Moraxella/Pasturella

Flavobacterium spp

Total

Grand total

21
16
11
48
93

Number of donors swabbed 782; plates with oxidase reactive colonies $124(15.9 \%)$; plates with oxidase positive Gram negative rods 93 (11.7\%); plates with Pseudomonas spp 8 (1.0\%); plates with Ps fluorescens $2(0.3 \%)$.

\section{Discussion}

The recent incidence of PTS both in the United Kingdom and the United States of America would indicate that it remains a potential risk of blood transfusion that should never be ignored.

Table 1, showing cases of PTS in the United Kingdom from 1980-1989, is unavoidably incomplete, because in some cases very few details are available. PTS is certainly underreported and the increase in the number of cases in 1987 could represent an increase in reporting as much as an increase in incidence.

During the 1980s, the USA experienced a similar upsurge in reported cases of PTS, so much so that in 1986 the American Association of Blood Banks, acting on the advice of the Food and Drug Administration, reduced the expiry date of platelet concentrates (which are stored at $22^{\circ} \mathrm{C}$ ) from seven to five days in an attempt to control this. ${ }^{8}$

PTS can be divided into two types: endogenous and exogenous, according to likely source of infection. In the former the organisms are circulating in the donor blood and are taken into the pack. Examples of this are cases involving Yersinia enterocolitica $a^{-13}$ and Salmonella spp $^{14}$ following gut infection. Also theoretically possible is the contamination of blood with Bacteroides spp and oral streptococci following gut or dental surgery.

Exogenous PTS includes cases in which contamination of the blood is from donor skin, or in which organisms gain entry via pack defects or contaminated equipment.

Endogenous PTS is difficult to avoid, other than by only accepting as donors those who conform to the general recommendations as laid down in United Kingdom Donor Selection Guidelines. ${ }^{15}$ However, inspection of packs for haemolysis before transfusion, attention to cleanliness of equipment such as centrifuges in case of inconspicuous pinhole defects and, most importantly, scrupulous arm cleansing of donors could minimise the incidence of exogenous PTS.

The bacterial types that comprise skin flora are probably taken into blood packs more often than is generally realised, but rarely cause fatal PTS because they cannot overcome the bacteriocidal effect of blood. ${ }^{1}$ Gram negative rods, however, especially Pseudomonas spp, may be transiently present on the donor skin. From here they can be taken into the blood pack (if the donor arm is inadequately cleaned) where they then may proliferate.

Since 1980 all reported cases of exogenous PTS have probably originated from donor skin contamination (there were two cases in the seventies that were shown to have other sources). ${ }^{1}$ In two cases, particularly since 1980 , skin contamination was strongly suspected, although not proved (A Murray, personal communication).

Although little can be done about endogenous PTS, exogenous PTS should be amenable to prevention. In this study, nearly $12 \%$ of donor arms tested yielded non-fermentative Gram negative rods. Pseudomonas spp were harboured by $1.0 \%$, and Ps fluorescens by $0.3 \%$. (table 2) Ps fluorescens, which has caused more known cases of PTS in this country since 1980 ( $60 \%$ in this study), may be found on the arms of about one donor in every 400 . Use of a skin cleansing procedure designed to eliminate this organism especially (as well as others) should therefore be mandatory. ${ }^{15}$

In the Oxford centre such a procedure has been routinely used since 1982 . It has been shown to clear the arm of skin flora, ${ }^{16}$ and in doing so, also removes any transient Gram negative rods such as Pseudomonas and Serratia spp. The procedure is monitored at weekly intervals by swabbing the arms of donors at a session before and immediately after cleansing (just before the needle is inserted). These swabs are then cultured on to heated blood agar and incubated at $30^{\circ} \mathrm{C}$ for 48 hours. The "after" plate should not yield any bacterial growth.

The advent of legislation concerning product liability has added renewed urgency to the awareness of transfusion transmitted infections, among which bacterial contamination of donations is an ever present risk. This study implicates Ps fluorescens as a potent cause of PTS and, although final proof is lacking, this organism probably usually originates from donor skin. It follows that universally applied effective donor skin cleansing is an obligatory requirement towards minimising the incidence of post transfusion septicaemia.

We thank Drs R Mitchell and A Napier for their helpful comments.

1 Puckett A. Bacterial contamination of blood for transfusion A study of the growth characteristics of four implicated organisms. Med Lab Sci 1986;43:252-7.

2 Tabor E, Gerety RJ. Five cases of Pseudomonas sepsis 
transmitted by blood transfusion. Lancet $1984 ; \mathrm{i}: 1403$.

3 Myre BA. Bacterial contamination is still a hazard of blood transfusion. Arch Pathol Lab Med 1985;109:983.

4 Braine HG, Kickler TS, Charache P, et al. Bacterial sepsis secondary to platelet transfusion: an adverse affect of extended storage at room temperature. Transfusion 1986; 26:391-3.

5 Murray AE, Bartzokas CA, Shepherd AJN, Roberts Freda $M$. Blood transfusion associated Pseudomonas fluorescens septicaemia: Is this an increasing problem? J Hosp Infect 1987;9:243-8.

6 Scott J, Bolton FE, Govan HRW, Miles RS, McClelland DBL, Prowse CV. A fatal transfusion reaction associated with blood contaminated with Pseudomonas fluorescens. Vox Sang 1988;54:201-4.

7 The Times (London) Youth died of blood transfusion. 1987; October 31.

8 Office of Biologics Research and Review, Department of Health and Human Services. Reduction of the maximum Health and Human Services. Reduction of the maximum
platelet storage period to 5 days in an approved container. platelet storage period to 5 days in an approved conte
9 Anonymous. Transfusion associated Yersinia enterocolitica. [Editorial]. Transfusion 1990;30:193-5.

10 Mitchell $R$, Barr A. Transfusion reaction due to Yersinia enterocolitica. Communicable Diseases Weekly Report. 1988;50:4.

11 Tipple MA, Bland LA, Murphy JJ, et al. Sepsis associated with Yersinia enterocolitica. Transfusion 1989;30:207.

12 Prentice M, Cope D, Weinbren M, O'Driscoll J. Infectious Complications of Blood Transfusion. BMJ 1990;300: 650.

13 Jacobs J, Jamaer D, Vandeven J, et al. Yersinia enterocolitica in donor Blood: a case report and review. $J$ Clin Microbiol 1989;27:1119-21.

14 Heal JM, Jones ME, Forey J, Chaudhry A, Stricof RL. Fatal Salmonella septicaemia after platelet transfusion. Transfusion 1987;27:2-5.

15 HSE/DSS. Guidelines for the Blood Transfusion Services in the United Kingdom Vol 1. London: HMSO, 1989:19-29, the United

16 Puckett A. A sterility method for testing blood products. Med Lab Sci 1986;43:249-51. 